Usef ul ness of $\mathrm{fI}$ uori ne- 18

$\mathrm{fl}$ uor odeoxygl ucose- posi tr on emi ssi on

tomogr aphy in management strategy for thym c epi thel i al t unors

\begin{tabular}{|l|l|}
\hline 著者 & $\begin{array}{l}\text { Nat sumbt o I sao, Oda Nakot o, Taki zawa Nasaya, } \\
\text { Waseda Ryui chi, Nakaj i ma Keni chi , Kawano } \\
\text { Nasaya, Mchi zuki Takaf um, I keda Hi roko, } \\
\text { Wat anabe Go }\end{array}$ \\
\hline $\begin{array}{l}\text { j our nal or } \\
\text { publ i cat i on t i t l e }\end{array}$ & Annal s of Thor aci c Sur ger y \\
\hline vol une & 95 \\
\hline nunber & 1 \\
\hline page r ange & $305-310$ \\
\hline year & $2013-01-01$ \\
\hline URL & ht t p: //hdl . handl e. net /2297/33405 \\
\hline
\end{tabular}




\title{
Usefulness of Fluorine-18 Fluorodeoxyglucose- Positron Emission Tomography in Management Strategy for Thymic Epithelial Tumors
}

\author{
Isao Matsumoto, MD, PhD, Makoto Oda, MD, $\mathrm{PhD}$, Masaya Takizawa, MD, PhD, \\ Ryuichi Waseda, MD, PhD, Kenichi Nakajima, MD, PhD, Masaya Kawano, MD, PhD, \\ Takafumi Mochizuki, MD, PhD, Hiroko Ikeda, MD, PhD, and Go Watanabe, MD, PhD
}

Departments of General and Cardiothoracic Surgery, and Nuclear Medicine/Biotracer Medicine, Kanazawa University; Kanazawa Advanced Medical Center, and Section of Diagnostic Pathology, Kanazawa University Hospital, Kanazawa, Japan

Background. This study investigated the usefulness of fluorine-18 fluorodeoxyglucose-positron emission tomography (FDG-PET) during the treatment of thymic epithelial tumors in combination with Ki-67 evaluation based on surgical cases in our department.

Methods. Between November 2003 and May 2011, 39 patients with thymic epithelial tumor underwent preoperative FDG-PET. The maximum standardized uptake value (SUVmax) of each category within Masaoka stage, World Health Organization classification, tumor diameter, myasthenia gravis, and Ki-67 label index were compared. To examine risk factors for relapse, SUVmax, age, sex, and surgical radicality were investigated in addition to those items.

Results. The mean SUVmax was 4.5 (range, 1.2 to 14.6) and was significantly higher for Masaoka stage IV than for I and II (all $p<0.008$ ) and for World Health Organization classified thymic cancer compared with all other types (all $p<0.0001$ ). Mean SUVmax revealed signifi-

$\mathrm{F}^{\circ}$ or the management strategy of thymic epithelial tumors, not only morphologic findings but also biologic features are important. Thymic epithelial tumors usually can be assessed with computed tomography (CT) and magnetic resonance imaging (MRI) [1, 2]. Morphologic features are used for characterizing thymic lesions, such as the contours and shapes of the lesions, the presence of necrosis, calcification, mediastinal fat or great vessel invasion, contrast enhancement, and adjacent lymph node enlargement. However, the morphologic findings of thymic lesions have many degrees of overlap among different entities, and thymic epithelial tumors show a broad spectrum of biologic and morphologic features $[3,4]$.

Fluorine-18 fluorodeoxyglucose $\left({ }^{18} \mathrm{~F}-\mathrm{FDG}\right)$ uptake on positron emission tomography (PET) has been used to discriminate between benign and malignant lesions in various organs. FDG-PET has emerged as a powerful

Accepted for publication Sept 13, 2012.

Address correspondence to Dr Matsumoto, Department of General and Cardiothoracic Surgery, Kanazawa University, 13-1 Takara-machi, Kanazawa 920-8641, Japan; e-mail: mat@p2223.nsk.ne.jp. cantly higher values for large tumors than for small tumors $(p=0.02)$. Mean SUVmax was significantly higher for high Ki-67-positive samples ( $p=0.0004)$, indicating a strong correlation between SUVmax and the Ki-67 label index ( $\rho=0.77, p=0.0001)$. SUVmax accurately reflected therapeutic efficacy in patients with induction therapy. Univariate analysis revealed Masaoka stages III and IV and pathologically incomplete resection as risk factors for relapse. On multivariate analysis, independent risk factors for relapse comprised only Masaoka stages III and IV.

Conclusions. FDG-PET SUVmax does reflect proliferation and invasiveness of thymic epithelial tumors and can provide an index for diagnosis and treatment, although it is not a risk factor for relapse. FDG-PET is also useful for evaluating induction therapy efficacy and detecting relapse.

(Ann Thorac Surg 2013;95:305-11) (C) 2013 by The Society of Thoracic Surgeons

diagnostic tool and is widely used for the diagnosis, staging, and restaging of various neoplasms. Also, it has been reported that FDG uptake on PET is correlated with tumor aggressiveness as assessed by proliferative activity and tumor invasiveness, particularly in lung tumors $[5,6]$. However, relatively few clinical studies with FDGPET have been conducted concerning thymic epithelial tumors, and the usefulness of FDG-PET scans for predicting histologic types and the evaluation of tumor aggressiveness of thymic epithelial tumors has not been examined adequately.

Meanwhile, Ki-67 is a widely used marker of proliferation and aggressiveness that has proven diagnostic and prognostic relevance in various solid tumors. A few studies have reported that the Ki-67 label index (LI) correlates with both the histology and invasiveness of thymoma [7, 8].

Only a few studies have reported the usefulness of FDG-PET in the management strategy of thymic epithelial tumors. The present study used data from surgical cases in our department to investigate the usefulness of FDG-PET during the treatment of thymic epithelial tumors combined with Ki-67 LI evaluation. 


\section{Patients and Methods}

The Kanazawa University Committee for Medical Ethics approved this retrospective study. Individual consent was obtained from the patients to use their resected specimen for research.

\section{Patients}

This study included 39 patients with thymic epithelial tumor who underwent preoperative FDG-PET. They were treated at our institution between November 2003 and May 2011. All patients underwent chest CT and FDG-PET preoperatively. Patients had to fast for 6 hours, and FDG-PET was performed only if their serum glucose level was less than $200 \mathrm{mg} / \mathrm{dL}$. Each patient was injected with $4.0 \mathrm{MBq} / \mathrm{kg}(0.11 \mathrm{mCi} / \mathrm{kg})$ of ${ }^{18} \mathrm{~F}-\mathrm{FDG}$. After an uptake of 60 minutes, patients were imaged using ECAT ACCEL (Siemens, Munich, Germany) until June 2007, and after that using a GE 4096 Plus PET whole body PET scanner (GE Medical System, Milwaukee, WI), from the base of the brain to the proximal thigh in 8 to 10 bed positions (early phase), followed by a delayed scan of the chest at 120 minutes (delayed phase). The maximum standardized uptake value (SUVmax) of the thymic lesion was determined by drawing regions of interest on the attenuation-corrected FDG-PET images around the thymic lesion. SUVmax was calculated using the software within the PET or PET-CT scanner and the following formula: SUVmax $=C(\mu \mathrm{Ci} / \mathrm{mL}) / \mathrm{ID}(\mu \mathrm{Ci}) / \mathrm{w}(\mathrm{kg})$, whereby $C$ is the activity at a pixel within the tissue defined by a region of interest, ID is the injected dose, and $w$ is the patient's body weight in kilograms. SUVmax within the selected regions of interest was used exclusively throughout this study.

This study compared the SUVmax of each category within Masaoka stage, World Health Organization (WHO) classification, tumor diameter, presence or absence of myasthenia gravis (MG), and Ki-67 LI. Furthermore, to examine risk factors for relapse, Masaoka stage (I/II as early stage or III/IV as advanced stage), WHO classification (A/AB/B1 as low-grade malignancy or B2/ $\mathrm{B} 3 /$ thymic cancer as high-grade malignancy), tumor diameter ( $\geq$ median or $<$ median), presence or absence of MG, Ki-67 LI ( $\geq$ median or $<$ median), SUVmax $(\geq$ median or $<$ median), age ( $\geq$ median or $<$ median), sex, and pathologically complete or incomplete resection were also investigated. Disease-free interval (DFI) was defined as the time from the resection of the tumor until relapse detected by CT or FDG-PET.

Ki-67 LI represents tumor cell proliferative activity and was immunohistologically evaluated using resected thymic epithelial tumor samples or preoperative biopsy samples if induction therapy (IT) was conducted. Proliferative activity was assessed immunohistochemically using the monoclonal antibody MIB1, which detects the proliferation-associated antigen Ki-67. Mouse monoclonal anti-human Ki-67 antigen (clone MIB1; Dako Corp, Glostrup, Denmark) was diluted 100-fold, and immunohistochemical staining was performed. The hematoxylin and eosin-stained slides of the tumor specimens were reviewed, and blocks at the edge of the tumor area were selected. Paraffin-embedded tumor tissues were cut into $4-\mu \mathrm{m}$-thick sections and deparaffinized. Immunohistochemical staining was performed using the labeled streptavidin-biotin method, as previously described [9]. Ki-67 LI was scored as the ratio of MIB1-stained tumor cells in which nuclear staining was positive [10, 11]. To assess Ki-67 LI, all fields of the sections were scanned at low (original magnification $\times 40$ ) and high (original magnification $\times 400$ ) power, and the most strongly stained areas were chosen. Evaluation of Ki-67 LI was performed using anonymous prepared slides to avoid identification of the patients.

For the pathologic evaluation of the IT treatment efficacy, we categorized the pathologic response according to the criterion of the General Rules of Clinical and Pathological Records of Lung Cancer (7th Edition) of Japan [12] which is based on the ratio of remaining viable cancer cells to the total cancer cells in a resected specimen. The criterion are Ef 0 , no pathologic changes; Ef $1 \mathrm{a}, 2 / 3$ and above; Ef 1 b, between 1/3 and 2/3; Ef 2, less than 1/3; Ef 3,0 . Two or more surgical pathologists reviewed the pathologic response of IT.

\section{Statistics}

The mean \pm standard deviation values are shown, and equality of means was analyzed with the unpaired $t$ test. Multiple comparisons of mean values between groups were drawn using the Tukey honestly significant difference test. The Pearson correlation test was used to investigate the relationship between SUVmax and Ki-67 LI in thymic epithelial tumors. Disease-free survival curves after resection of thymic epithelial tumors were calculated using the Kaplan-Meier method and compared using the log-rank test. Univariate and multivariate Cox proportional hazards model was used to examine the risk factors of relapse and to estimate hazard ratios (HR) and confidence interval (CI). Values of $p$ of less than .05 were considered significant. Analyses were performed with PASW Statistics 18 software (SPSS In., Chicago, IL).

\section{Results}

There were 16 men and 23 women, with a mean age of 55 years (range, 26 to 81 years). The mean maximum diameter of thymic tumor was $61.5 \mathrm{~mm}$ (range, 20 to $137 \mathrm{~mm}$ ). The number of patients classified as Masaoka stages I, II, III, or IV were $4,22,8$, and 5 , and those classified WHO type $\mathrm{A}, \mathrm{AB}, \mathrm{B} 1, \mathrm{~B} 2, \mathrm{~B} 3$, or thymic cancer were $1,4,13,11$, 7 and 3, respectively. Eight patients were complicated by MG, and 5 underwent IT. For IT, patients basically received a unified multiagent chemotherapeutic regimen including cisplatin and docetaxel. Three patients underwent neoadjuvant chemotherapy plus concurrent radiation at a dose of $40 \mathrm{~Gy}$. Operative procedures comprised extended thymothymectomy in 8 with combined resection of surrounding organs in 2, and thymothymectomy resection in 31 with combined resection of surrounding organs in 13. Complete resection was confirmed in 34 
SUVmax

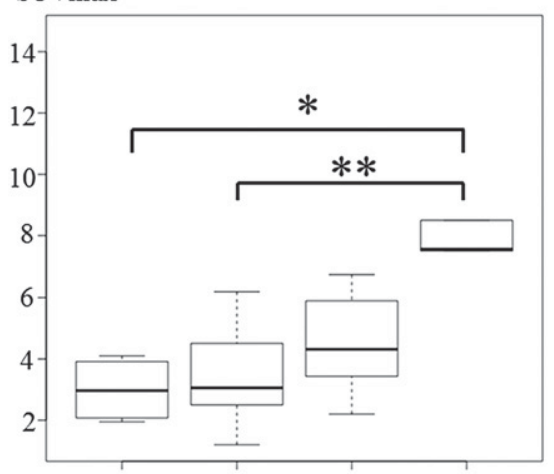

$\Delta$ I $\quad$ II $\quad$ III IV
SUVmax

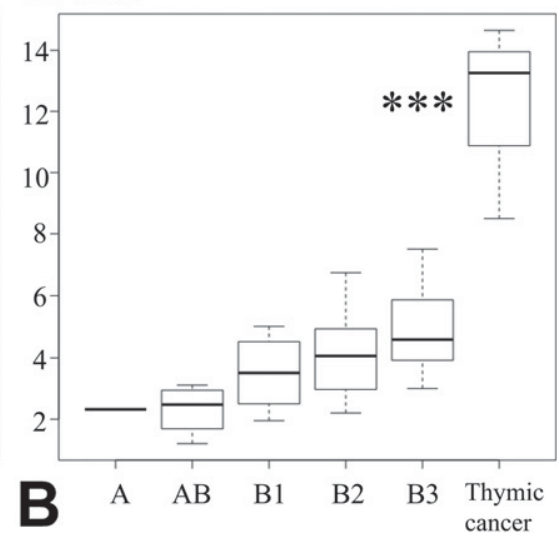

Fig 1. Box and whisker plots show comparison of mean maximum standardized uptake value (SUVmax) of each category within (A) Masaoka stage and (B) within the World Health Organization (WHO) classification. For WHO classification, SUVmax of thymic cancer was higher compared with all other types. The horizontal line in the middle of each box indicates the median, the top and bottom borders of the box mark the 75th and 25 th percentiles, respectively, and the whiskers mark the 90th and 10th percentiles. ${ }^{*} p<0.008 ;{ }^{* *} p<0.001 ;{ }^{* * *} p<0.001$ (significant difference). patients; however, 5 had pathologically incomplete resection.

The operative-related mortality rate was $0 \%$. Postoperative complications included 1 patient each with postoperative crisis of MG, chylothorax, and pleural effusion. Major cardiovascular, pulmonary, gastrointestinal, and technical morbidity did not occur.

The mean follow-up after the initial operation of thymic lesion was 40 months (range, 4 to 104; median, 58 months). All patients were monitored with periodic CT and FDG-PET. Seven patients relapsed, and recurrent lesion was confirmed in all by FDG-PET confirmed recurrent lesion.

The mean SUVmax of the tumors in FDG-PET was 4.5 (range, 1.2 to 14.6; median, 3.9). Early and delayed SUVs were measured in 10 patients, and mean SUVmax tended to be higher in delayed images than in early images, at $5.9 \pm 4.6$ and $4.9 \pm 3.9$, respectively $(p=0.62)$.

The mean value of each Masaoka stage and each type of WHO classification are shown in Figure 1. The SUVmax of patients classified as Masaoka stages I, II, III, or IV were $3.0 \pm 1.1,3.6 \pm 1.4,5.3 \pm 3.4$, and $8.3 \pm 4.0$ and WHO types A, AB, B1, B2, B3, or thymic cancer were 2.3, $2.3 \pm 0.8,3.7 \pm 1.5,4.1 \pm 1.4,4.9 \pm 1.5$, and $12.1 \pm 3.2$. Mean SUVmax was significantly higher for Masaoka stage IV than for stages I and II (all $p<0.008$ ) and for
WHO classification thymic cancer compared with all other types (all $p<0.0001$ ). SUVmax tended to be higher for advanced stage and higher malignant grade tumors.

Comparison of mean SUVmax between large (diameter $\geq$ median) and small (diameter $<$ median) tumors revealed significantly higher values for large tumors, $5.6 \pm 3.3$ and $3.5 \pm 1.8$, respectively ( $p=0.02$, Fig $2 \mathrm{~A})$. Mean SUVmax tended to be higher in patients without MG (absence, $4.9 \pm 3.0$; presence, $3.0 \pm 0.9 ; p=0.09$; Fig 2B).

Positive staining for Ki-67 was set at $30 \%$ or higher (median value), and mean SUVmax was significantly higher for positive samples (positive, $6.0 \pm 3.2$; negative, $3.0 \pm 0.9 ; p=0.0004 ;$ Fig 3A), indicating a strong correlation between SUVmax and Ki-67 LI $(\rho=0.77, p=$ 0.0001; Fig 3B).

The Ki-67 LI of patients classified as Masaoka stages I, II, III, or IV were $0.20 \pm 0.09,0.28 \pm 0.17,0.36 \pm 0.18$, and $0.54 \pm 0.30$, and for WHO types $\mathrm{A}, \mathrm{AB}, \mathrm{B} 1, \mathrm{~B} 2, \mathrm{~B} 3$, or thymic cancer were $0.16,0.11 \pm 0.10,0.25 \pm 0.15,0.34 \pm$ $0.13,0.39 \pm 0.15$, and $0.78 \pm 0.13$, respectively. Mean Ki-67 LI tended to be higher for Masaoka stage IV than for stages I $(p=0.058)$ and II $(p=0.056)$ and for WHO classification thymic cancer compared with all other types (all $p<0.004)$ and B3 compared with $\mathrm{AB}(p=0.04)$.

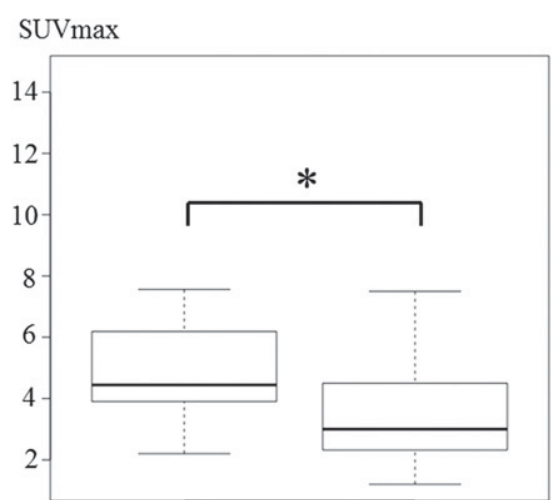

A tumor diameter tumor diameter $\geq 60 \mathrm{~mm}$ $<60 \mathrm{~mm}$
SUVmax

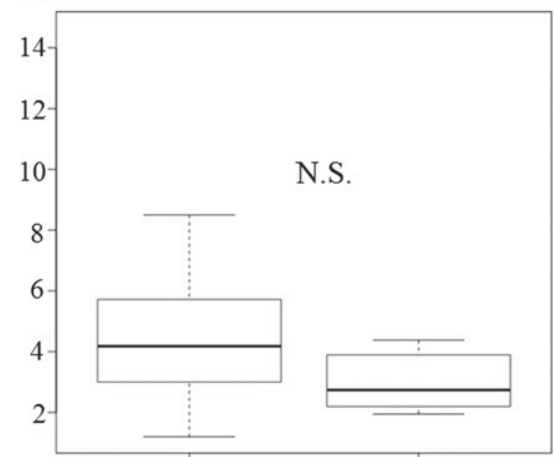

B myasthenia gravis myasthenia gravis

Fig 2. Box and whisker plots show comparison of mean maximum standardized uptake value (SUVmax) between (A) large (diameter $\geq$ median) and small (diameter $<$ median) tumors and between (B) patients with and without myasthenia gravis. The horizontal line in the middle of each box indicates the median, the top and bottom borders of the box mark the 75th and 25th percentiles, respectively, and the whiskers mark the 90th and 10th percentiles. ${ }^{*} p<0.02$ (significant difference). (N.S. = not significant.) 
Fig 3. (A) Box and whisker plot shows comparison of mean maximum standardized uptake value (SUVmax) between high Ki-67 label index ( $\geq$ median) and low Ki-67 label index $(<$ median). The horizontal line in the middle of each box indicates the median, the top and bottom borders of the box mark the 75 th and 25th percentiles, respectively, and the whiskers mark the 90th and 10th percentiles. (B) Correlation is shown between SUVmax and Ki-67 label index. ${ }^{*} p<0.0004$ (significant difference).

Ki-67 LI also tended to be higher for advanced-stage tumors and those of higher malignant grade.

Table 1 reports the pathologic evaluation of treatment efficacy and changes of SUVmax between pre-IT and post- IT in the 5 IT patients. SUVmax accurately reflected therapeutic efficacy, with increased treatment efficacy on pathologic evaluation associated with larger decreases in mean SUVmax.

The univariate analysis showed Masaoka stages III and IV (advanced stage) and pathologically incomplete resection were risk factors for relapse (Table 2). On multivariate analysis, independent risk factors for relapse comprised only Masaoka stages III and IV (Table 3).

\section{Comment}

According to the results of this study, FDG-PET SUVmax tended to be higher for advanced-stage tumors and those at a higher malignant grade with a large amount of epithelial components, especially in thymic cancer. In addition, because a strong correlation between SUVmax and Ki-67 LI was confirmed, SUVmax was demonstrated to reflect proliferation and invasiveness and can provide an index for diagnosis and treatment. SUVmax is also useful for evaluating IT efficacy and detecting relapse; thus, even if the thymic epithelial tumors do not shrink after IT, the decision for a surgical or nonsurgical treat- ment can be made based on FDG-PET findings. However, SUVmax is not a risk factor for relapse of thymic epithelial tumors.

Thymic epithelial tumors are common primary neoplasms in the anterior mediastinum and are usually slow-growing tumors, except for thymic cancer. However, they include variable histologic features and heterogeneous oncologic behavior. Histologic features include neoplastic epithelial cells with differing shapes and degrees of atypia and various degrees of nonneoplastic lymphoid cells in the tumors. FDG-PET scanning is performed to obtain further biologic information about the lesions. Since Liu and colleagues [13] reported in the first study on the role of FDG-PET in the detection of thymoma, only about a dozen publications to date have reported FDG-PET imaging for thymic lesions.

Liu [4] reviewed these studies and summarized that PET-CT is effective in differentiating thymic cancer from other entities within the thymus and thymoma from thymic hyperplasia, although the results for differentiation between invasive and noninvasive thymomas may be more equivocal. In those series, thymic cancer showed a significantly higher SUV than thymoma and thymic hyperplasia [4, 14]. The previous reports indicated that the classification of thymomas by FDG-PET correlated with the clinical Masaoka stage rather than the WHO classification, and a high SUV usually reflected invasive-

Table 1. Positron Emission Tomography Maximum Standardized Uptake Value Change and Induction Therapy Effectiveness ${ }^{a}$

\begin{tabular}{lcccccccc}
\hline & \multicolumn{2}{c}{ Masaoka } & & & \multicolumn{2}{c}{ SUVmax } \\
\cline { 2 - 3 } Patient & c-Stage & p-Stage & WHO Classification & Treatment & Pre-IT & Post-IT & IT Effects \\
\hline 1 & IVa & IVa & B1 & CR & 7.5 & 1.5 & Ef $1 b$ \\
2 & III & II & B3 & CR & 5.5 & 0.5 & Ef 2 \\
3 & IVa & IVa & B1 & Chemotherapy & 2.0 & 1.0 & Ef 1a \\
4 & IVb & IVb & B3 & CR & 7.5 & 2.3 & Ef 1b \\
5 & III & III & B2 & Chemotherapy & 6.8 & 6.9 & Ef 0 \\
\hline
\end{tabular}

${ }^{a}$ IT effectiveness was evaluated by the ratio of remaining viable cancer cells in a resected specimen. $\quad{ }^{b}$ Ef 0 , no pathologic changes; Ef $1 \mathrm{a}, 2 / 3$ and above; Ef $1 b$, between 1/3 and 2/3; Ef 2, less than 1/3. ${ }^{\mathrm{C}}$ Innominate vein invasion.

c-Stage = clinical Masaoka stage; $\quad \mathrm{CR}=$ chemotherapy and radiotherapy; $\quad \mathrm{IT}=$ induction therapy; $\mathrm{p}$-Stage $=$ pathological Masaoka stage; SUVmax = maximum standardized uptake value; $\quad$ WHO = World Health Organization. 
Table 2. Univariate Analysis of Risk Factors for Relapse

\begin{tabular}{|c|c|c|c|}
\hline Factor & $\begin{array}{l}\text { Patients } \\
\text { (No.) }\end{array}$ & $\begin{array}{c}\mathrm{HR} \\
(95 \% \mathrm{CI})\end{array}$ & $\begin{array}{c}p \\
\text { Value }\end{array}$ \\
\hline Masaoka stage & & $0.243(0.055-0.592)$ & 0.001 \\
\hline \multicolumn{4}{|l|}{ Early stage } \\
\hline I & 4 & & \\
\hline II & 22 & & \\
\hline \multicolumn{4}{|l|}{ Advanced stage } \\
\hline III & 8 & & \\
\hline IV & 5 & & \\
\hline WHO classification & & $0.417(0.096-1.009)$ & 0.053 \\
\hline \multicolumn{4}{|c|}{ Low-grade malignancy } \\
\hline A & 1 & & \\
\hline $\mathrm{AB}$ & 4 & & \\
\hline B1 & 13 & & \\
\hline \multicolumn{4}{|c|}{ High-grade malignancy } \\
\hline B2 & 11 & & \\
\hline B3 & 7 & & \\
\hline Cancer & 3 & & \\
\hline Tumor diameter & & $0.791(0.351-1.687)$ & 0.538 \\
\hline$<60 \mathrm{~mm}$ & 21 & & \\
\hline$\geq 60 \mathrm{~mm}$ & 18 & & \\
\hline Myasthenia gravis & & $0.716(0.164-1.742)$ & 0.506 \\
\hline Presence & 8 & & \\
\hline Absence & 31 & & \\
\hline Ki-67 label index & & $0.572(0.216-1.234)$ & 0.158 \\
\hline$<30 \%$ & 20 & & \\
\hline$\geq 30 \%$ & 19 & & \\
\hline SUVmax & & $0.496(0.187-1.072)$ & 0.075 \\
\hline$<4.0$ & 20 & & \\
\hline$\geq 4.0$ & 19 & & \\
\hline Age, years & & $0.768(0.359-1.735)$ & 0.499 \\
\hline$<60$ & 25 & & \\
\hline$\geq 60$ & 14 & & \\
\hline Sex & & $0.764(0.289-1.646)$ & 0.506 \\
\hline Male & 16 & & \\
\hline Female & 23 & & \\
\hline Surgical radicality & & $0.353(0.164-0.799)$ & 0.015 \\
\hline Complete & 34 & & \\
\hline Incomplete & 5 & & \\
\hline
\end{tabular}

$\mathrm{CI}=$ confidence interval; $\quad \mathrm{HR}=$ hazard ratio; $\quad \mathrm{SUVmax}=$ maxi mum standardized uptake value; WHO $=$ World Health Organization.

ness of a malignant nature by the thymic tumor, whereas a low SUV reflected a less invasive malignant thymoma [15]. In contrast, Ito and colleagues [16] argued that the low or high level of the SUV is a reflection of thymomas of type B1 or of the combined types B1 plus B2, respec- tively, and that the classification of thymomas by FDGPET probably correlates not only with the clinical stage but also with the WHO histologic subtype. Our results showed that it is not possible to specify Masaoka stages or the WHO histologic subtype with the absolute value of SUVmax; however, advanced Masaoka stages and WHO malignant grade subtypes had high SUVmax.

Our results showed a strong correlation between SUVmax and Ki-67 LI. To our knowledge, this is the first report that demonstrates such relationship in thymic epithelial tumors. Many studies have correlated Ki-67 expression with clinical outcome in malignant tumors. It is clear that Ki-67 is of prognostic value for many types of malignant tumors [17]. The effectiveness of using Ki-67 antibody as a tool to measure proliferative activity has been widely investigated in a variety of tumors, however, rarely in thymic epithelial tumors. The study by Luebke and colleagues [17] using Ki-67 found that the Masaoka staging system may be of prognostic relevance in thymoma type B3 and thymic cancer, and Ki-67 may indicate the malignant potential of thymic carcinoma. As described in our study, Ki-67 and SUVmax reflected proliferation and invasiveness of thymic epithelial tumor; however, the value did not correlate significantly with risk factors for relapse of the tumor. Independent risk factors for relapse comprised only Masaoka stages III and $\mathrm{IV}$, as found in other studies [17, 18]. Kondo and colleagues [18] showed in a recent clinical study of 1,320 patients with thymic epithelial tumors that the Masaoka clinical stage was an excellent predictor of the prognosis of thymoma and thymic carcinoma, but not of thymic carcinoid.

In the present study, we found that SUVmax accurately reflected therapeutic efficacy in the IT patients, with increased treatment efficacy on pathologic evaluation associated with larger decreases in mean SUVmax. Morphologic imaging cannot reliably distinguish a postoperative scar or necrosis from a residual or recurrent tumor. Response evaluation with morphologic imaging does not correlate well with the pathologic response. The metabolic response criteria seem to be more sensitive and accurate. Kaira and colleagues [19] also reported that PET-CT was useful in monitoring and predicting response and outcome after treatment in unresectable thymic epithelial tumors. In addition, we confirmed recurrent lesions by FDG-PET in all relapsed patients.

Early detection and exact localization of tumor recurrence are important for the planning of further therapy. Occasionally, CT scans cannot differentiate residual and recurrent disease from fibrosis and scar tissue, especially when the disease is located in the anterior mediastinum

Table 3. Multivariate Analysis of Risk Factors for Relapse

\begin{tabular}{lllcc}
\hline Factor & Favorable & Unfavorable & HR (95\% CI) & $p$ Value \\
\hline Masaoka stage & I and II & III and IV & $0.283(0.062-0.795)$ & 0.016 \\
Surgical radicality & Complete & Incomplete & $0.690(0.295-1.612)$ & 0.372 \\
\hline
\end{tabular}

$\mathrm{CI}=$ confidence interval; $\quad \mathrm{HR}=$ hazard ratio. 
at a previous surgical site. FDG-PET is sensitive and specific in diagnosing residual disease. Thus, PET is also useful in the follow-up of patients who have undergone thymic epithelial tumor excision when recurrence or metastasis is suspected [16, 19, 20].

Some investigators reported the usefulness of FDGPET but have also observed a marked overlap in the uptake of noninvasive and invasive thymomas [4, 14]. In those reports, similarly to our results, SUVmax was proportional to the size of the tumor. Small tumors are not necessary noninvasive, however. We note that partial-volume effects can cause a marked underestimation of the true activity concentration within a tumor [21]. For a spheric lesion with a diameter equivalent to 1.5 times the spatial resolution of the PET scanner at full width half maximum, the measured maximum activity concentration is only about $60 \%$ the true activity concentration. The mean activity concentration is even lower, about $30 \%$ the true activity concentration. Only when the diameter of the lesion is about four times higher than the spatial resolution of the scanner is the difference between the measured peak activity concentration and the true activity concentration less than 5\% [22]. The measured mean activity concentration is still only approximately $60 \%$ the true activity concentration [21].

Our analysis has some limitations. This study is a retrospective examination of a small series of patients with thymic epithelial tumors surgically treated at a single institution. Although thymomas are included in the entity of malignancy, they have varied degree of malignancy and there are few relapses or metastases compared with cancer due to their slow-growing behavior. Therefore, we could not evaluate the effect of SUVmax on the prognosis of the patients with thymic epithelial tumors because of short follow-up periods. To clearly establish the utility of this method, further studies with a greater number of patients and long-term follow-up periods are needed. In addition, to assess Ki-67 LI, limited areas with small amount of lymphocytes were scanned in WHO type B1 and B2 thymomas. Because lymphocytes are easily stained by Ki-67, it is difficult to detect Ki-67 stained tumor cells in lymphocytesrich tumors.

In conclusion, PET SUVmax does reflect proliferation and invasiveness of thymic epithelial tumor and can provide an index for diagnosis and treatment, although it is not a risk factor for relapse. This value is also useful for evaluating IT efficacy and detecting relapse. Independent risk factors for relapse comprised only Masaoka stages III and IV.

\section{References}

1. Jeong YJ, Lee KS, Kim J, Shim YM, Han J, Kwon OJ. Does CT of thymic epithelial tumors enable us to differentiate histologic subtypes and predict prognosis? AJR Am J Roentgenol 2004;183:283-9.

2. Inaoka T, Takahashi K, Mineta M, et al. Thymic hyperplasia and thymus gland tumors: differentiation with chemical shift MR imaging. Radiology 2007;243:869-76.
3. Restrepo CS, Pandit M, Rojas IC, et al. Imaging findings of expansile lesions of the thymus. Curr Probl Diagn Radiol 2005;34:22-34.

4. Liu Y. Characterization of thymic lesions with F-18 FDG PET-CT: an emphasis on epithelial tumors. Nucl Med Commun 2011;32:554-62.

5. Vesselle H, Schmidt RA, Pugsley JM, et al. Lung cancer proliferation correlates with [F-18]fluorodeoxyglucose uptake by positron emission tomography. Clin Cancer Res 2000;6:3837-44.

6. Nomori H, Watanabe K, Ohtsuka T, et al. Fluorine 18-tagged fluorodeoxyglucose positron emission tomographic scanning to predict lymph node metastasis, invasiveness, or both, in clinical T1 N0 M0 lung adenocarcinoma. J Thorac Cardiovasc Surg 2004;128:396-401.

7. Raica M, Mogoantă L, Kondylis A, Cîmpean AM. Angiogenesis in the human thymoma assessed by subclassification of tumor-associated blood vessels and endothelial cells proliferation. Rom J Morphol Embryol 2010;51:627-31.

8. Pan CC, Ho DM, Chen WY, Huang CW, Chiang H. Ki67 labelling index correlates with stage and histology but not significantly with prognosis in thymoma. Histopathology 1998;33:453-8.

9. Ohta Y, Nozawa H, Tanaka Y, Oda M, Watanabe Y. Increased vascular endothelial growth factor and vascular endothelial growth factor-c and decreased $\mathrm{nm} 23$ expression associated with microdissemination in the lymph nodes in stage I non-small cell lung cancer. J Thorac Cardiovasc Surg 2000;119:804-13.

10. Cooper LS, Gillett CE, Smith P, Fentiman IS, Barnes DM. Cell proliferation measured by MIB1 and timing of surgery for breast cancer. Br J Cancer 1998;77:1502-7.

11. Bottini A, Berruti A, Bersiga A, et al. Relationship between tumour shrinkage and reduction in Ki67 expression after primary chemotherapy in human breast cancer. $\mathrm{Br} \mathrm{J}$ Cancer 2001;85:1106-12.

12. The Japan Lung Cancer Society. General rules of clinical and pathological records of lung cancer. 7th ed [in Japanese]. Tokyo: Kanehara; 2010;175-7.

13. Liu RS, Yeh $\mathrm{SH}$, Huang $\mathrm{MH}$, et al. Use of fluorine-18 fluorodeoxyglucose positron emission tomography in the detection of thymoma: a preliminary report. Eur J Nucl Med 1995;22:1402-7.

14. Sung YM, Lee KS, Kim BT, Choi JY, Shim YM, Yi CA. 18F-FDG PET/CT of thymic epithelial tumors: usefulness for distinguishing and staging tumor subgroups. J Nucl Med 2006; 47:1628-34.

15. Kubota K, Yamada S, Kondo T, et al. PET imaging of primary mediastinal tumours. Br J Cancer 1996;73:882-6.

16. Ito $\mathrm{H}$, Shimada $\mathrm{K}$, Isogami $\mathrm{K}$, et al. Recurrent thymoma: radiological (CT and FDG-PET) and histological (WHO criteria) features. Radiat Med 2006;24:292-6.

17. Luebke T, Beckurts KT, Wickenhauser C, Schneider P, Hoelscher AH, Moenig SP. Masaoka staging is of prognostic relevance in type B3/C thymomas. Anticancer Res 2004;24: 4113-9.

18. Kondo K, Monden Y. Therapy for thymic epithelial tumors: a clinical study of 1,320 patients from Japan. Ann Thorac Surg 2003;76:878-84.

19. Kaira K, Murakami H, Miura S, et al. 18F-FDG uptake on PET helps predict outcome and response after treatment in unresectable thymic epithelial tumors. Ann Nucl Med 2011; 25:247-53.

20. El-Bawab H, Al-Sugair AA, Rafay M, Hajjar W, Mahdy M, Al-Kattan K. Role of flourine-18 fluorodeoxyglucose positron emission tomography in thymic pathology. Eur J Cardiothorac Surg 2007;31:731-6.

21. Weber WA. Use of PET for monitoring cancer therapy and for predicting outcome. J Nucl Med 2005;46:983-95.

22. Geworski L, Knoop BO, de Cabrejas ML, Knapp WH, Munz DL. Recovery correction for quantitation in emission tomography: a feasibility study. Eur J Nucl Med 2000;27:161-9. 